\title{
DENSE EXTREMALLY DISCONNECTED SUBSPACES
}

\author{
A. DOW AND J. vaN MILL
}

(Communicated by Franklin D. Tall)

\begin{abstract}
We prove that every compact Basically Disconnected space of $\pi$ weight $\omega_{1}$ has a dense Extremally Disconnected subspace. In Boolean algebraic terms: every $\sigma$-complete Boolean algebra $B$ with density $\omega_{1}$ carries an ultrafilter which generates an ultrafilter in the completion of $B$. The statement that every compact Basically Disconnected space of weight $c$ has a dense Extremally Disconnected subspace is shown to be equivalent to $\mathbf{C H}$.
\end{abstract}

\section{INTRODUCTION}

Three closely related classes of zero-dimensional spaces are the Extremally Disconnected (the clopen algebra is complete), abbreviated ED, the Basically Disconnected (the clopen algebra is $\sigma$-complete), abbreviated BD, and the $P$ spaces (the clopen algebra is closed under countable unions and intersections). The question of when certain spaces have dense $P$-spaces has been extensively studied. We are looking for dense Extremally Disconnected subspaces. As can be seen from the discussion below, for zero-dimensional spaces this is the same as finding ultrafilters on Boolean algebras which generate ultrafilters on the completion.

A point in a space is a $\lambda$-point (for a cardinal $\lambda$ ) if there are $\lambda$ disjoint open sets each with the point in the closure. A space is ED if and only if no point of the space is a 2-point. Call a point which is not a 2-point an ED-point.

A point $p \in \beta X \backslash X$ is called a remote point of $X$ if $p$ is not in the closure of any nowhere dense subset of $X$. Close connections have been established between remote points, ED spaces, and 2-points. For example, Woods [Woo71] established that the set of remote points of a space $X$ embeds homeomorphically (and canonically) into the (ED) Gleason space of $\beta X$. More detailed connections between remote points and 2-points are explored in van Douwen's paper [vD81]. In particular, every remote point of $X$ is an ED-point of $\beta X$. The converse is false because if $X$ is ED then $\beta X$ is ED at every point. It is easily seen that a space has a dense ED subspace if and only if it has a dense set of points which are not 2-points.

Received by the editors October 23, 1991.

1991 Mathematics Subject Classification. Primary 54G05, 54A35.

Key words and phrases. Extremally and Basically Disconnected, remote points, Boolean algebra.

The first author thanks the Department of Mathematics at Vrije Universiteit for generous hospitality and support. 
In the first section we prove that every compact BD space of $\pi$-weight $\omega_{1}$ has a dense ED subspace. This is somewhat surprising because the usual way to construct ED-points, i.e., via remote points, does not work here; indeed, it follows trivially from Dow [Dow83] that if $\mathbf{C H}$ fails there is a BD space with $\pi$-weight $\omega_{1}$ which has no remote points. We also construct an example of a compact BD space with weight $\max \left\{\omega_{2}, \mathfrak{c}\right\}$ and $\pi$-weight $\omega_{2}$ in which every point is a 2-point. Therefore the statement that every compact $\mathrm{BD}$ space of weight $c$ has a dense ED subspace is equivalent to $\mathbf{C H}$. It has already been shown that the BD space consisting of the uniform ultrafilters on $\omega_{1}$ (which has weight $2^{\omega_{1}}$ ) does not have a dense ED space (see Balcar and Simon [BaSi82]).

\section{COMPACT BD SPACES OF $\pi$-WEIGHT $\aleph_{1}$ HAVE DENSE ED SUBSPACES}

Recall that a $\pi$-base for a space $X$ is a collection $\mathscr{B}$ of nonempty open subsets of $X$ such that every nonempty open subset of $X$ contains a member of $\mathscr{B}$. The $\pi$-weight, $\pi(X)$, of $X$ is the smallest cardinality of a $\pi$-base for $X$. In the proof of the following result we will make use of ideas in Chae and Smith [CS80] and van Douwen [vD81].

Theorem 2.1. Every compact BD space with $\pi(X) \leq \omega_{1}$ has a dense ED subspace.

Proof. Let $X$ be a compact BD space with $\pi$-base $\mathscr{B}=\left\{B_{\alpha}: \alpha<\omega_{1}\right\}$. We may assume without loss of generality that $\mathscr{B}$ consists of clopen sets. Let $\mathscr{W}$ be the family of all nonempty open subsets of $X$. For each $W \in \mathscr{W}$ put

$$
H(W)=\left\{\alpha<\omega_{1}: B_{\alpha} \subset W \text { or } B_{\alpha} \cap \bar{W}=\varnothing\right\} .
$$

Note that $\bar{W}-W$ is nowhere dense, for $W \in \mathscr{W}$, hence $\left\{B_{\alpha}: \alpha \in H(W)\right\}$ is again a $\pi$-base for $X$. For $W \in \mathscr{W}$ set $\mu(W)<\omega_{1}$ to be a nonzero ordinal with the property that

$$
(\forall \beta<\mu(W))(\exists \alpha<\mu(W)) B_{\alpha} \subseteq B_{\beta} \text { and } \alpha \in H(W) .
$$

The following closing off argument shows that there is such an ordinal $\mu(W)$ :

$$
\begin{aligned}
\mu(W, 1) & =\min H(W), \\
\mu(W, m+1) & =\min \left\{\alpha<\omega_{1}:[\forall \beta \leq \mu(W, m)][\exists \xi \in H(W) \cap \alpha]\left[B_{\xi} \subseteq B_{\beta}\right]\right\} .
\end{aligned}
$$

Then $\mu(W)=\sup \{\mu(W, m): 1 \leq m<\omega\}$ has the desired property.

For each $W \in \mathscr{W}$ let $U_{W}=\bigcup\left\{B_{\alpha}: \alpha \in H(W) \cap \mu(W)\right\}$, note that $U_{W}$ is an open $F_{\sigma}$.

Fact 2.2. If $\mathscr{E} \in[\mathscr{W}]^{<\omega}$ then there is an $\alpha<\max \{\mu(W): W \in \mathscr{E}\}$ such that $B_{\alpha} \subseteq \bigcap_{W \in \mathscr{E}} U_{W}$.

We will prove the fact by induction on $n=|\mathscr{E}|$. For $n=1$, it is obvious from the definition of $\mu(W)$. So assume the fact to be true for $n$, and consider arbitrary $W_{1}, \ldots, W_{n+1} \in \mathscr{W}$. We may assume that for all $i \leq n+1$ we have $\mu\left(W_{i}\right) \leq \mu\left(W_{n+1}\right)$. By our inductive hypothesis, there is an $\alpha<\max \left\{\mu\left(W_{i}\right): 1 \leq i \leq n\right\}$ such that $B_{\alpha} \subseteq \bigcap_{i=1}^{n} U_{W_{i}}$. Since $\alpha<\mu\left(W_{n+1}\right)$ there is $\beta<\mu\left(W_{n+1}\right)$ with $B_{\beta} \subseteq B_{\alpha}$ and $\beta \in H\left(W_{n+1}\right)$. Then $B_{\beta} \subseteq \bigcap_{i=1}^{n+1} U_{W_{i}}$ and $\beta<\max \left\{\mu\left(W_{i}\right): i \leq n+1\right\}$, so we are done.

We conclude that in particular the collection $\left\{U_{W}: W \in \mathscr{W}\right\}$ has the finite intersection property. So by compactness of $X$ we may pick a point $x$ in $\bigcap_{W \in \mathscr{W}} \bar{U}_{W}$. 
Fact 2.3. $x$ is not a 2-point.

To the contrary, assume that $x$ is a 2-point. Then there is an open $W$ of $X$ such that

$$
x \in \bar{W} \quad \text { and } \quad x \in \overline{X-\bar{W}} \text {. }
$$

Put $A=U_{W} \cap W$ and $B=U_{W}-\bar{W}$, respectively. Observe that both these sets are open $F_{\sigma}$ 's since $A=\bigcup\left\{B_{\beta}: \beta<\mu(W)\right.$ and $\left.B_{\beta} \subset W\right\}$ and $B=\bigcup\left\{B_{\beta}: \beta<\right.$ $\mu(W)$ and $\left.B_{\beta} \cap \bar{W}=\varnothing\right\}$. Now $A$ is disjoint from the open set $X-\bar{W}$, hence so is $\bar{A}$. Since $\bar{A}$ is clopen it is also disjoint from $\overline{X-\bar{W}}$. Therefore $x$ cannot be a member of $\bar{A}$. Similarly $\bar{B}$ is disjoint from $\bar{W}$, hence $x$ is not a member of $\bar{B}$. However this contradicts that $x \in \overline{U_{W}} \subset \bar{A} \cup \bar{B}$.

We conclude that $X$ is somewhere ED. But the same reasoning can be applied to every nonempty clopen subspace of $X$. So it follows that $X$ contains a dense ED subspace.

Observe that the above proof can be generalized. If $X$ is a compact zerodimensional space of $\pi$-weight $\kappa$ such that the union of fewer than $\kappa$ clopen sets has clopen closure, then $X$ has a dense ED subspace.

Corollary 2.4. If $\mathbf{C H}$ holds then every compact BD space of weight $\mathfrak{c}$ contains a dense ED subspace.

Two questions naturally arise. Is $\mathbf{C H}$ (or its full strength) needed to prove Corollary 2.4? Can the hypothesis $\pi(X) \leq \omega_{1}$ be (consistently) weakened in Theorem 2.1? In fact, only the parenthetical questions are open. Indeed, Balcar and Simon have shown that $U\left(\omega_{1}\right)$ does not have a dense ED subspace [BaSi82]. This space is BD and has $\pi$-weight at most $2^{\omega_{1}}$. In the next section we prove that both 2.1 and 2.4 are best possible in the sense that the statement in Corollary 2.4 is equivalent to $\mathbf{C H}$.

\section{A sMall BD sPaCe With No DENSE ED subsPaCe}

In this section a $\sigma$-complete Boolean algebra $\mathfrak{U}$ of cardinality $\omega_{2} \cdot \mathfrak{c}$ is constructed whose Stone space is shown to have the property that every point is a 2-point, hence there is no dense ED subspace.

For every space $X$, let $R O(X)$ denote the complete Boolean algebra of regular open subsets of $X$. If $\mathscr{A} \subseteq R O(X)$ then its supremum in $R O(X)$ is int $\operatorname{cl}(\bigcup \mathscr{A})$. For more information on $R O(X)$ we refer the reader to Porter and Woods [PW88].

Notation 3.1. For an $s \in{ }^{<\omega} \omega_{2}$, let $[s]=\left\{x \in{ }^{\omega} \omega_{2}: s \subset x\right\}$. In addition, put

$$
\mathfrak{S}=\left\{[s]: s \in{ }^{<\omega} \omega_{2}\right\} \text {. }
$$

For convenience we treat members of ${ }^{<\omega} \omega_{2}$ as ordered sequences of ordinals. In particular, $s^{\frown} \gamma$ denotes the obvious extension of $s$.

We endow $\omega_{2}$ with the discrete topology and ${ }^{\omega} \omega_{2}$ with the product topology, i.e., the topology having $\mathfrak{S}$ as a basis. Observe that if $[s]$ and $[t]$ are in $\mathfrak{S}$ and if $[s] \cap[t] \neq \varnothing$ then $[s] \subseteq[t]$ or $[t] \subseteq[s]$.

Definition 3.2. Let $U \in R O\left({ }^{\omega} \omega_{2}\right)$. Then $\mathscr{A}_{U}$ is the collection of all countable sets $A_{U} \subseteq \omega_{2}$ such that for any $s \in{ }^{<\omega} \omega_{2}$, either $U \cap[s \frown \gamma]$ is empty for all

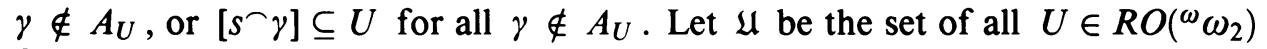
for which $\mathscr{A}_{U} \neq \varnothing$. 
Proposition 3.3. $\mathfrak{U}$ is a $\sigma$-complete subalgebra of $R O\left({ }^{\omega} \omega_{2}\right)$ that contains $\mathfrak{S}$. Proof. It is immediate that $\mathfrak{U}$ is closed under complements (in $R O\left({ }^{\omega} \omega_{2}\right)$ ). Suppose that $\left\{U_{n}: n \in \omega\right\} \subset \mathfrak{U}$ and let $U=\bigvee_{n \in \omega} U_{n}=\operatorname{int} \operatorname{cl}\left(\bigcup_{n \in \omega} U_{n}\right)$. For each $n$, let $A_{U_{n}}$ witness that $U_{n}$ is in $\mathfrak{U}$ and let $A=\bigcup_{n} A_{U_{n}}$. Fix any $s \in{ }^{<\omega} \omega_{2}$ and let $\gamma \notin A$. Suppose that $U \cap\left[s^{\sim} \gamma\right] \neq \varnothing$. Then there is an $n$ such that $U_{n} \cap\left[s^{\frown} \gamma\right] \neq \varnothing$. It follows that $\left[s^{\wedge} \delta\right] \subset U_{n}$ for all $\delta \notin A_{U_{n}}$. Therefore $[s \neg \delta] \subset U$ for all $\delta \notin A$, hence $U \in \mathfrak{U}$.

It is routine to check that $\mathfrak{U} \supseteq \mathfrak{S}$.

It will be useful to find an explicit description of the elements of $\mathfrak{U}$.

Remark 3.4. Let us emphasize that if $U \in \mathfrak{U}$ and $s \in{ }^{<\omega} \omega_{2}$ and if for some $\gamma_{0} \notin A_{U} \in \mathscr{A}_{U},\left[s^{\frown} \gamma_{0}\right] \cap U \neq \varnothing$ then $\left[s^{\frown} \gamma\right] \subseteq U$ for every $\gamma \notin A_{U}$.

If $U \in \mathfrak{U}$ then we put $B_{U}=\bigcap \mathscr{A}_{U}$. We will show that $B_{U} \in \mathscr{A}_{U}$ (see Corollary 3.10). Define a function $\varphi_{U}:{ }^{<\omega}\left(B_{U}\right) \rightarrow 2$ as follows:

$$
\varphi_{U}(s)=1 \Leftrightarrow\left(\forall \gamma \notin B_{U}\right)\left(\left[s^{\wedge} \gamma\right] \subseteq U\right) .
$$

Proposition 3.5. Let $U \in \mathfrak{U}$. Then

$$
\bigcup\left\{\left[s^{\frown} \gamma\right]: s \in{ }^{<\omega}\left(B_{U}\right), \gamma \notin B_{U} \text {, and } \varphi_{U}(s)=1\right\}
$$

is dense in $U$.

Proof. Suppose that $V=\bigcup\left\{\left[s^{\frown} \gamma\right]: s \in \epsilon^{<\omega}\left(B_{U}\right), \gamma \notin B_{U}\right.$, and $\left.\varphi_{U}(s)=1\right\}$ is not dense in $U$. Pick a nonempty $[t] \in \mathfrak{S}$ such that $[t] \subseteq U \backslash V$. We may assume without loss of generality that the last element of $t$ is not in $B_{U}$ (because if it is, then we can replace $t$ by $t^{\frown} \gamma$ for an arbitrary $\gamma \notin B_{U}$ ). Let $\gamma$ be the first member of $t$ that does not belong to $B_{U}$. Write $t$ in the form $t=t_{0} \frown \gamma^{\frown} t_{1}$. Observe that $t_{0} \in{ }^{<\omega}\left(B_{U}\right)$.

Claim 3.6. $\varphi_{U}\left(t_{0}\right)=1$.

Pick an arbitrary $\delta \notin B_{U}$. There exist elements $A_{U}, \widehat{A}_{U} \in \mathscr{A}_{U}$ such that $\delta \notin A_{U}$ and $\gamma \notin \hat{A}_{U}$. Since $\varnothing \neq[t] \subseteq\left[t_{0}-\gamma\right] \cap U$, it follows by Remark 3.4 that for cocountably many $\xi$ we have $\left[t_{0}-\xi\right] \subseteq U$. Now if $\left[t_{0}-\delta\right]$ were not contained in $U$ then it would follow again by Remark 3.4 that for cocountably many $\eta$ we have $\left[t_{0} \frown \eta\right] \cap U=\varnothing$; this is clearly impossible. So we conclude that $\left[t_{0} \frown \delta\right] \subseteq U$.

Since $\varphi_{U}\left(t_{0}\right)=1$ and $\gamma \notin B_{U}$ it now follows that $\left[t_{0} \frown \gamma\right] \subseteq V$. But this clearly contradicts the fact that $[t]$ and $V$ do not intersect because $[t] \subseteq$ $\left[t_{0} \frown \gamma\right]$.

Corollary 3.7. Let $U \in \mathfrak{U}$ and $s \in{ }^{<\omega}\left(B_{U}\right)$ and let $B=B_{U}$. Then

$$
\varphi_{U}(s)=0 \Leftrightarrow(\forall \gamma \notin B)\left(\left[s^{\frown} \gamma\right] \cap U=\varnothing\right) .
$$

Proof. Suppose that $\varphi_{U}(s)=0$. Pick an arbitrary $\gamma$ not in $B$, and assume that $\left[s^{\sim} \gamma\right] \cap U \neq \varnothing$. By Proposition 3.5 there exists $t \in{ }^{<\omega} B$ with $\varphi_{U}(t)=1$ and $\gamma_{1} \notin B$ such that $\left[s^{-} \gamma\right] \cap\left[t^{\sim} \gamma_{1}\right] \neq \varnothing$. Consequently, $\left[s^{\sim} \gamma\right] \subseteq\left[t^{\sim} \gamma_{1}\right]$ or $\left[t^{\sim} \gamma_{1}\right] \subseteq\left[s^{\sim} \gamma\right]$. Since both $s$ and $t$ belong to ${ }^{<\omega} B$ and both $\gamma$ and $\gamma_{1}$ do not belong to $B$, it follows that $s=t$ and $\gamma=\gamma_{1}$. But this is a contradiction because $\varphi_{U}(s)=0$ and $\varphi_{U}(t)=1$.

To every $U \in \mathfrak{U}$ we assigned a function $\varphi_{U}$. We now aim to show that this function completely determines $U$. 
Lemma 3.8. If $U, V \in \mathfrak{U}$, and $U \neq V$ then $\varphi_{U} \neq \varphi_{V}$.

Proof. We may assume that the domains of $\varphi_{U}$ and $\varphi_{V}$ agree. Since $U \neq V$ by Proposition 3.5 we may also assume without loss of generality that there exists $s \in{ }^{<\omega}\left(B_{U}\right)$ with $\varphi_{U}(s)=1$ and $\gamma \notin B_{U}$ such that $\left[s^{-} \gamma\right]$ intersects the complement of $V$. But since the domains of $\varphi_{U}$ and $\varphi_{V}$ agree, this implies that $\varphi_{V}(s)=0$ because otherwise $\left[s^{\sim} \gamma\right] \subseteq V$.

Since for each countable $B$ there are $c$ functions from $B$ into 2 and there are $\omega_{2}^{\omega}=\mathfrak{c} \cdot \omega_{2}$ countable subsets of $\omega_{2}$, this lemma implies that $|\mathfrak{U}| \leq \mathfrak{c} \cdot \omega_{2}$.

Proposition 3.9. If $B \subset \omega_{2}$ is countable and $\varphi:{ }^{<\omega} B \rightarrow 2$ is any function, then

$$
U=\operatorname{intcl}\left(\bigcup\left\{\left[s^{\frown} \gamma\right]: s \in{ }^{<\omega} B, \gamma \notin B \text {, and } \varphi(s)=1\right\}\right)
$$

is in $\mathfrak{U}$ and $B \in \mathscr{A}_{U}$.

Proof. We prove that $B \in \mathscr{A}_{U}$. To this end, pick any arbitrary $t \in \mathfrak{S}$. Assume that there exists $\gamma \notin B$ such that $\left[t^{-} \gamma\right] \cap U \neq \varnothing$. Pick $s \in{ }^{\omega} B$ with $\varphi(s)=1$ and an element $\gamma_{1} \notin B$ such that $\left[t^{\wedge} \gamma\right] \cap\left[s^{\sim} \gamma_{1}\right] \neq \varnothing$. Then $\left[t^{\wedge} \gamma\right] \subseteq\left[s^{\sim} \gamma_{1}\right]$ or $\left[s^{\sim} \gamma_{1}\right] \subseteq\left[t^{\sim} \gamma\right]$. If $t^{\frown} \gamma$ is an initial sequence of $s^{\sim} \gamma_{1}$ then since $\gamma \notin B$ it follows that $t=s$ and $\gamma=\gamma_{1}$. But then $\left[t^{-} \delta\right] \subseteq U$ for every $\delta \notin B$. Suppose therefore that $s^{\frown} \gamma_{1}$ is an initial sequence of $t^{\frown} \gamma$. We may assume without loss of generality that $\gamma_{1}$ comes before $\gamma$ because otherwise we are again in the situation that $s=t$. So $s^{\sim} \gamma_{1}$ is an initial sequence of $t$, which implies that every extension of $t$ is contained in $\left[s^{\frown} \gamma_{1}\right] \subseteq U$.

Corollary 3.10. Let $U \in \mathfrak{U}$. Then $B_{U} \in \mathscr{A}_{U}$.

Proof. This follows easily from Propositions 3.5 and 3.9.

Although we do not need it, let us remark that $\mathfrak{U}$ is the smallest $\sigma$-complete subalgebra of $R O\left({ }^{\omega} \omega_{2}\right)$ that contains $\mathfrak{S}$.

We have shown above that the cardinality of $\mathfrak{U}$ is no more than $\omega_{2} \cdot \mathfrak{c}$. It also contains $\mathfrak{S}$ which has cardinality $\omega_{2}$. Since the cardinality of $\mathfrak{U}$ is an $\omega$ power by the result of Comfort and Hager [CH72] it follows that its cardinality is $\omega_{2} \cdot \mathfrak{c}$. This also follows easily from Proposition 3.9.

We will proceed to prove that each point of $S(\mathfrak{U})$ is a 2-point.

Definition 3.11. For each $\alpha<\omega_{2}$, let $E$ be the set of even ordinals in $\omega_{2}$ and $O=\omega_{2} \backslash E$, and define

$$
W_{\alpha}=\bigcup\left\{[s]:(\exists n)\left(s(n) \in E \backslash \alpha \wedge s \mid n \in{ }^{n} \alpha\right)\right\}
$$

and

$$
V_{\alpha}=\bigcup\left\{[s]:(\exists n)\left(s(n) \in O \backslash \alpha \wedge s \mid n \in{ }^{n} \alpha\right)\right\} .
$$

We now come to the main result in this section.

Theorem 3.12. Every point of the Stone space of $\mathfrak{U}$ is a 2-point.

Proof. Let $p$ be an arbitrary ultrafilter on $\mathfrak{U}$ and assume that it is not a 2-point. Let $S(\mathfrak{U})$ denote the Stone space of $\mathfrak{U}$. Observe that for every $\alpha$ both $W_{\alpha}$ and $V_{\alpha}$ are unions of elements of $\mathfrak{U}$; moreover, $W_{\alpha} \cap V_{\alpha}=\varnothing$. So the sets $W_{\alpha}$ and $V_{\alpha}$ correspond in a natural way to disjoint open subsets, say $W_{\alpha}^{*}$ and $V_{\alpha}^{*}$, of $S(\mathfrak{U})$. Observe that $p$ is in the closure of $W_{\alpha}^{*}$ if and only if $U \cap W_{\alpha} \neq \varnothing$ for every $U \in p$. Similarly for $V_{\alpha}^{*}$. 
So, since $p$ is not a 2-point, there is, for each $\alpha<\omega_{2}$, a $U_{\alpha} \in p$ such that $U_{\alpha}$ is disjoint from either $W_{\alpha}$ or $V_{\alpha}$. By definition of $\mathfrak{U}$ there is, for each $\alpha \in \omega_{2}, A_{\alpha} \in \mathscr{A}_{U_{\alpha}}$. Choose an increasing sequence, $\left\{\lambda_{\xi}: \xi \in \omega_{1}\right\}$, so that $A_{\lambda_{\xi}} \subset \lambda_{\xi+1}$ for each $\xi<\omega_{1}$. Let $\lambda_{\omega_{1}}$ be the supremum of the sequence $\left\{\lambda_{\xi}: \xi \in \omega_{1}\right\}$. Choose $\alpha<\omega_{1}$ so that $A_{\omega_{\omega_{1}}} \cap \lambda_{\omega_{1}} \subseteq \lambda_{\alpha}$. Let $s \in{ }^{<\omega} \omega_{2}$ be such that [s] is contained in $U_{\lambda_{\alpha}} \cap U_{\lambda_{\omega_{1}}}$. Since, without loss of generality, $U_{\lambda_{\alpha}} \cap W_{\lambda_{\alpha}}$ is empty, there is a minimum $k$ such that $s(k) \geq \lambda_{\alpha}$; else any extension of $s$ by a sufficiently large even ordinal witnesses that $U_{\lambda_{\alpha}}$ meets $W_{\alpha}$.

We will show that $s(k) \in A_{\lambda_{\alpha}} \cap A_{\lambda_{\omega_{1}}}$, which is a contradiction since $A_{\lambda_{\alpha}} \cap$ $A_{\lambda_{\omega_{1}}} \subseteq \lambda_{\omega_{1}} \cap A_{\lambda_{\omega_{1}}} \subseteq \lambda_{\alpha}$, and yet $s(k) \geq \lambda_{\alpha}$.

We first prove by contradiction that $s(k) \in A_{\lambda_{\alpha}}$. Observe that $\left[s\lceil k] \cap U_{\lambda_{\alpha}} \neq\right.$ $\varnothing$. Apply Remark 3.4 to see that for all but countably many $\gamma,\left[\left(s\lceil k)^{-} \gamma\right]\right.$ is contained in $U_{\lambda_{\alpha}}$. But now since $s \mid k \in{ }^{<\omega} \lambda_{\alpha}$ it follows that some of these clopen sets are contained in $W_{\lambda_{\alpha}}$.

The proof that $s(k) \in A_{\lambda_{\omega_{1}}}$ is identical.

\section{REFERENCES}

[BaSi82] B. Balcar and P. Simon, Strong decomposability of ultrafilters I, Studies in Logic, vol. 108, North-Holland, Amsterdam, 1980, pp. 1-10.

[CS80] S. B. Chae and J. H. Smith, Remote points and G-spaces, Topology Appl. 11 (1980), 243-246.

[CH72] W.W. Comfort and T. Hager, Cardinality of $\kappa$-complete Boolean algebras, Pacific J. Math. 28 (1972), 541-545.

[vD81] E. K. van Douwen, Remote points, Dissertationes Math. 188 (1981), 1-45.

[Dow83] A. Dow, Products without remote points, Topology Appl. 15 (1983), 239-246.

[PW88] J. Porter and R. G. Woods, Extensions and absolutes of Hausdorff spaces, Springer-Verlag, New York, Heidelberg, and Berlin, 1988.

[Woo71] R. G. Woods, Homeomorphic sets of remote points, Canad. J. Math. 23 (1971), 495-502.

Department of Mathematics, York University, Toronto, Ontario, Canada M3J 1P3

E-mail address: DOWACNEXUS. YORKU.CA

Department of Mathematics and Computer Science, VRije Universiteit, De BoelelaAn 1081 A, 1081 HV AMSTERdaM, THE NetherLandS

E-mail address: VANMILL@CS.VU.NL 